\title{
Fatores prognósticos da cirurgia de Latarjet
}

\section{Prognostic Factors of the Latarjet Procedure}

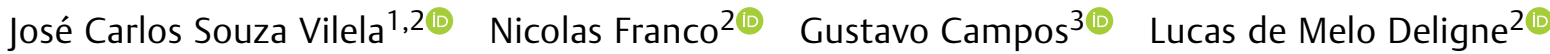

Thalles Leandro Abreu Machado 2,3 (1)

1 Hospital Mater Dei, Belo Horizonte, MG, Brasil
2 Hospital Unimed BH, Belo Horizonte, MG, Brasil
3 Ortopedia e Traumatologia, Instituto de Previdência dos Servidores
do Estado de Minas Gerais (Ipsemg), Belo Horizonte, MG, Brasil

\begin{abstract}
Endereço para correspondência Thalles Leandro Abreu Machado, MD, Rua Grão Pará, 658, $2^{\circ}$ andar, Santa Efigênia, Belo Horizonte, MG, 30150-341, Brasil

(e-mail: thallescirurgiadoombro@gmail.com/www.imotbh.com.br).
\end{abstract}

Rev Bras Ortop 2022;57(6):975-983.

\section{Resumo \\ Palavras-chave \\ - luxação do ombro/cirurgia \\ - ombro/cirurgia \\ - instabilidade articular \\ - lesões de Bankart \\ - qualidade de vida \\ Objetivo Investigar os fatores que influenciam os resultados funcionais dos pacientes submetidos ao procedimento de Latarjet. \\ Métodos Avaliação de 26 pacientes submetidos ao tratamento cirúrgico, seguindo a técnica de Latarjet, devido a luxação anterior recidivante traumática da articulação glenoumeral, com perda óssea glenoidal maior do que $20 \%$ e/ou lesão off-track. O tempo mínimo de seguimento foi de doze meses. Foram avaliadas as seguintes escalas: Escala Visual Analógica (EVA), The Western Ontario Shoulder Instability Index (WOSI), e Subjective Shoulder Value (SSV), além dos dados objetivos dos participantes. \\ Resultados A maioria dos pacientes (84,62\%) não apresentou recidiva de luxação, e $92,31 \%$ ficaram satisfeitos. Em relação à análise funcional, a pontuação do componente físico (PCF) e a pontuação do compenente mental (PCM) encontradas estavam dentro da média de qualidade de vida da população. Os sintomas físicos, pela escala WOSI, apresentaram a melhor porcentagem (8,5\%), ao passo que o pior resultado foi observado com relação ao estilo de vida (20\%). Na EVA, a dor foi classificada como moderada (3/10) por $15,38 \%$ dos pacientes (4/26). Em relação aos esportes, os pacientes que praticavam alguma atividade esportiva apresentaram melhora nos parâmetros da escala SSV, que tiveram relação inversa com o número de recidivas. Observou-se ainda que, quanto menor foi o tempo entre a primeira luxação e a realização da cirurgia, maior foi a satisfação do paciente. \\ Conclusão A indicação precoce do tratamento cirúrgico da instabilidade anterior glenoumeral pode proporcionar melhores resultados funcionais subjetivos ao paciente.}

\footnotetext{
* Trabalho desenvolvido no Hospital Unimed BH, Belo Horizonte, MG, Brasil.
}

recebido

15 de Dezembro de 2020

aceito

08 de Julho de 2021

Publicado on-line

Novembro 11, 2021
DOI https://doi.org/

10.1055/s-0041-1736513. ISSN 0102-3616. (c) 2021. Sociedade Brasileira de Ortopedia e Traumatologia. All rights reserved.

This is an open access article published by Thieme under the terms of the Creative Commons Attribution-NonDerivative-NonCommercial-License, permitting copying and reproduction so long as the original work is given appropriate credit. Contents may not be used for commercial purposes, or adapted, remixed, transformed or built upon. (https://creativecommons.org/ licenses/by-nc-nd/4.0/)

Thieme Revinter Publicações Ltda., Rua do Matoso 170, Rio de Janeiro, RJ, CEP 20270-135, Brazil 


Abstract


Keywords
- shoulder
dislocation/surgery
- shoulder/surgery
- joint instability
- Bankart injuries
- quality of life

Objective To investigate the factors that influence the functional results of patients submitted to the Latarjet procedure.

Methods Evaluation of 26 patients submitted to surgical treatment following the Latarjet technique due to traumatic recurrent anterior dislocation of the glenohumeral joint, with glenoidal bone loss greater than $20 \%$ and/or off-track injury. The minimum follow-up time was of 12 months. The Visual Analogue Scale (VAS), The Western Ontario Shoulder Instability Index (WOSI), and the Subjective Shoulder Value (SSV) scales, as well as objective data from the participants, were evaluated.

Results Most patients (84.62\%)did not present recurrence of the dislocation, and 92.31\% were satisfied. Regarding the functional analysis, the physical component score (PCS) and the mental component score (MCS) found were within the mean quality of life of the population. The physical symptoms, according to the WOSI, presented the best percentage (8.5\%), while the worst result was observed regarding lifestyle (20\%). On the VAS, pain was classified as moderate $(3 / 10)$ by $15.38 \%$ of the patients $(4 / 26)$. In relation to sports, patients who practice sports showed improvement in SSV parameters, which had an inverse relationship with the number of relapses. It was also observed that the shorter the time between the first dislocation and the surgery, the greater the patient's satisfaction.

Conclusion Early indication of surgical treatment of anterior glenohumeral instability may provide better subjective functional results to the patient.

\section{Introdução}

Diferentes procedimentos cirúrgicos foram propostos para o tratamento da luxação anterior recidivante traumática do ombro. O reparo capsulolabial foi descrito por Bankart e modificado por Rowe et al., ${ }^{1}$ e pode ser realizado por método cirúrgico aberto ou artroscópico. Em um estudo cadavérico, Itoi et al. $^{2}$ demonstraram que defeitos ósseos da glenoide maiores do que $21 \%$ diminuem a força necessária para causar a luxação, e recomendaram enxertia óssea para aumentar a estabilidade.

Em 1954, Latarjet ${ }^{3}$ descreveu a transferência do processo coracoide com fixação na porção anterior da glenoide, que produz dois efeitos: o enxerto do coracoide atuando como bloqueio ósseo, e o tendão conjunto com efeito tipoia atuando como bloqueio dinâmico. Tradicionalmente, o procedimento é realizado por via aberta, mas alguns autores recentemente passaram a defender a via artroscópica. $^{4,5}$

0 procedimento de Latarjet apresenta taxas de recidiva menores do que as dos reparos aberto ou artroscópico de Bankart. $^{6-8}$ Em uma revisão sistemática, Bliven e Parr ${ }^{9}$ demonstraram também melhores resultados funcionais e menor limitação da rotação externa com o procedimento de Latarjet quando comparado à cirurgia de Bankart. Contudo, apresenta maiores taxas de complicações, como pseudartrose, complicações com o material de síntese, e lesões neurovasculares associadas. ${ }^{10-12}$

$\mathrm{Na}$ avaliação de retorno ao esporte, tanto a cirurgia de Latarjet quanto a de Bankart não demonstram diferenças, apesar de haver poucos estudos comparativos. ${ }^{13-15}$
O objetivo deste estudo é investigar os fatores que influenciam os resultados funcionais nos pacientes submetidos ao procedimento de Latarjet. A hipótese é a de que tanto o atraso do paciente em procurar atendimento quanto a demora para o retorno ao esporte após o procedimento afetam diretamente a sua qualidade de vida e a satisfação.

\section{Pacientes e Métodos}

O estudo foi aprovado pelo Comitê de Ética em Pesquisa de nossa instituição. Foi solicitada e aprovada a dispensa do termo de consentimento livre e esclarecido (TCLE) para o projeto.

Trata-se de um estudo com uma coorte retrospectiva, realizado no período de julho de 2014 a julho de 2018, em que 41 pacientes foram submetidos ao tratamento cirúrgico de luxação anterior traumática recidivante do ombro pelo procedimento aberto de Latarjet, em um hospital particular de nível terciário. Os pacientes foram operados por dois cirurgiões de ombro participantes do estudo como pesquisadores, sendo que ambos utilizaram a mesma técnica.

Os critérios de inclusão foram: pacientes com luxação anterior recidivante traumática da articulação glenoumeral, maiores de 18 anos, submetidos ao procedimento cirúrgico de Latarjet por via aberta, com lesões off-track com perda óssea glenoidal maior do que 20\% (-Figura 1 ) - aferida pelo método no qual se comparam as distâncias entre o bare spote as bordas anterior e posterior da glenoide -, e com seguimento pós-operatório mínimo de 1 ano. Os critérios de exclusão foram: pacientes com luxação anterior não traumática da articulação glenoumeral, menores de 18 anos, com 


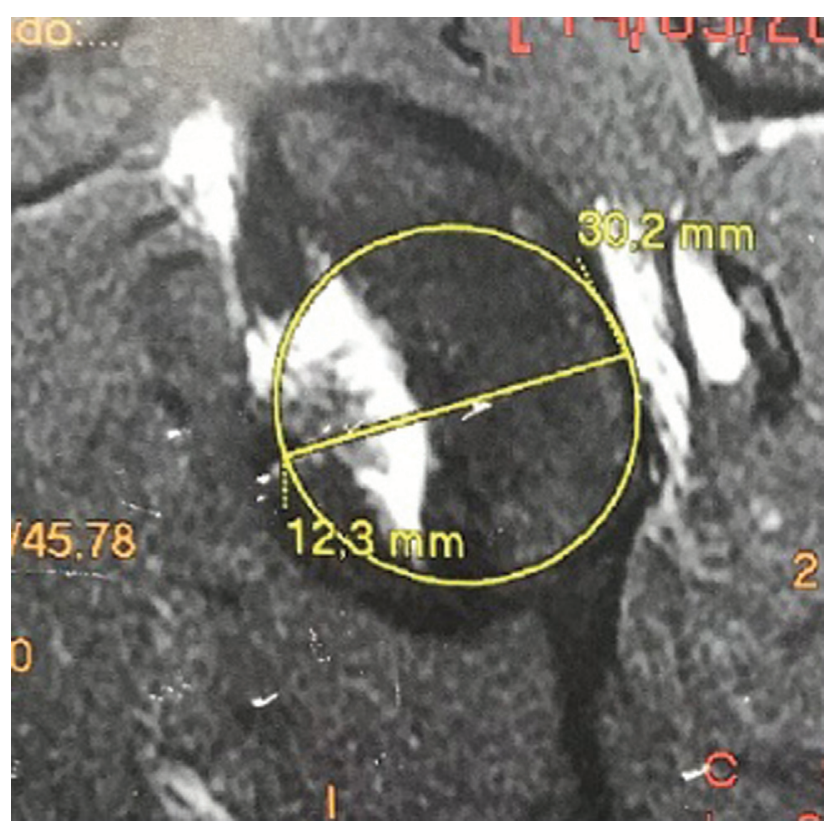

Fig. 1 Imagem de ressonância magnética no corte sagital ponderada em T2, que mostra defeito ósseo glenoidal.

lesões on-track, e perda óssea menor do que $20 \%$. No pósoperatório, os pacientes usaram tipoia por quatro semanas. Na quarta semana, iniciou-se a fisioterapia com atividades leves no plano horizontal, com ganho progressivo de amplitude de movimento (ADM). No terceiro mês, iniciaram-se exercícios de fortalecimento. $O$ retorno às atividades normais ocorreu após 5 a 6 meses de pós-operatório.

Após a consideração de todos os critérios mencionados, a amostra constituiu-se de 26 pacientes que responderam ao questionário e participaram da pesquisa, sendo que 3haviam sido submetidos ao procedimento cirúrgico previamente. 0 contato feito por telefone, assim como foi respondido o questionário. Não se conseguiu contato com 15 pacientes.
Por conta da pandemia de doença do coronavírus 2019 (coronavirus disease 2019, COVID-19, em inglês), houve dificuldade para avaliar presencialmente os pacientes no ano de 2020.

\section{Técnica Cirúrgica}

Os pacientes foram submetidos a bloqueio do plexo braquial para analgesia pós-operatória e anestesia geral, e foram operados em posição de cadeira de praia. Utilizou-se o acesso deltopeitoral, expôs-se o processo coracoide, e fez-se a transecção do ligamento coracoacromial e do músculo peitoral menor, protegendo-se os nervos musculocutâneo e axilar. Em seguida, mediu-se o enxerto do coracoide, em justaposição aos ligamentos coracoclaviculares com uma angulação de $90^{\circ}$ e, na direção medial para a lateral, com o destacamento do enxerto. 0 enxerto foi então preparado com a decorticação de sua face inferior, com o auxílio de uma lâmina de shaver. Com o ombro em rotação externa, o músculo subescapular foi exposto e dividido entre os dois terços superiores e o terço inferior com uma tesoura Mayo. A cápsula glenoumeral anterior foi identificada e incisada transversalmente para a exposição da glenoide. Realizouse a ressecção do tecido labial anteroinferior da glenoide, e o local de fixação do enxerto, na face anteroinferior do colo da glenoide, foi preparado e cruentizado. 0 processo coracoide foi colocado na posição entre 3 e 5 horas dos ponteiros do relógio, e a fixação foi realizada com 2 parafusos esponjosos de pequenos fragmentos (de 3,5 mm) na direção anteroposterior, perpendicular ao coracoide e paralelo à superfície articular da glenoide ${ }^{16}$ (-Figura 2). 0 membro operado foi colocado em uma tipoia Vealpeau simples. O curativo foi trocado no primeiro dia de pós-operatório, e os pacientes fizeram as trocas subsequentes diariamente em suas residências até o dia da retirada dos pontos, na segunda semana de pós-operatório. 0 membro operado foi imobilizado com a tipoia por quatro semanas, sendo que os movimentos de

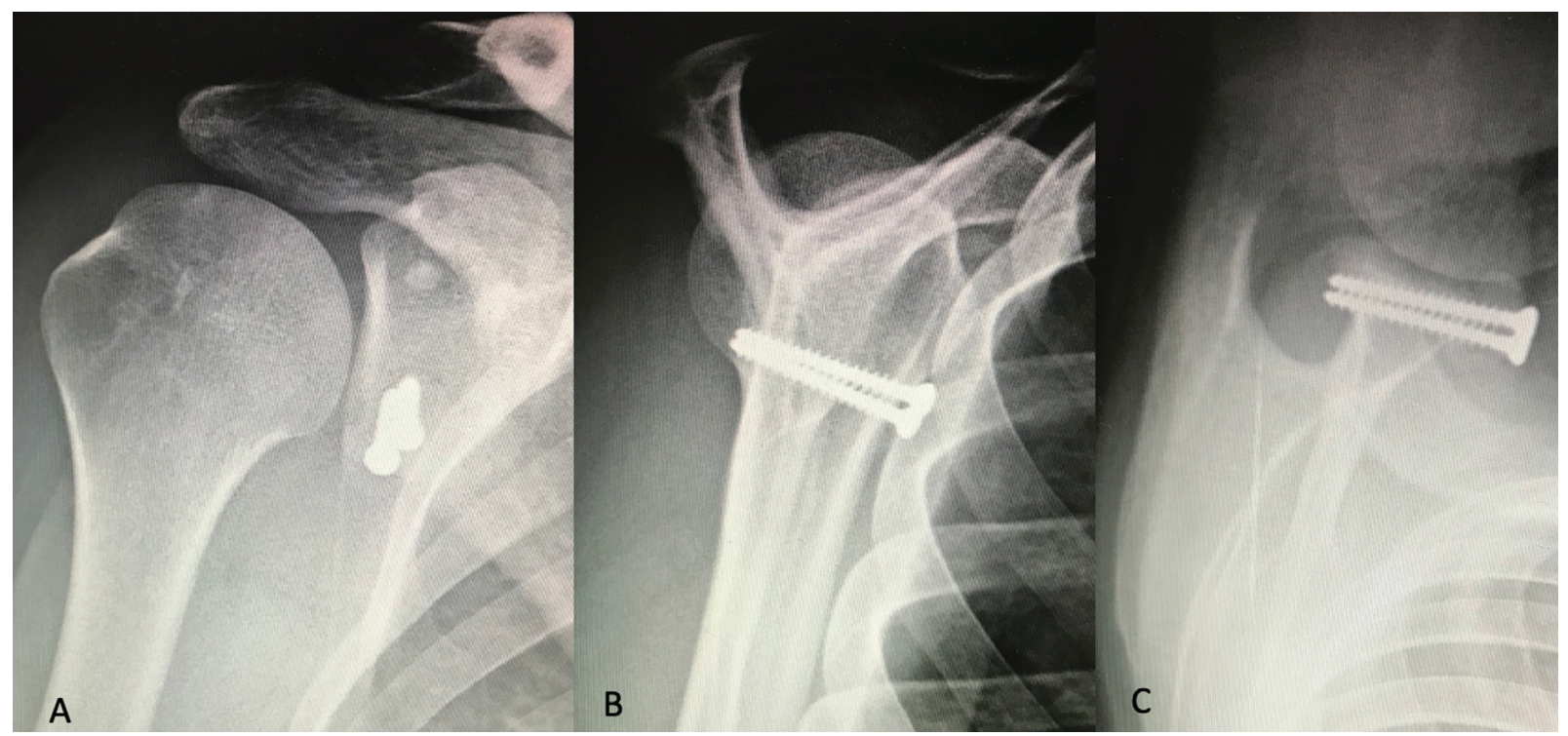

Fig. 2 Radiografias que mostram a fixação com parafusos esponjosos na ciruriga de Latarjet: (A) incidência anteroposterior; (B) incidência de perfil; (C) incidência axilar. 
flexão e extensão do cotovelo foram iniciados no primeiro dia de pós-operatório. A partir da quarta semana, foi iniciada a fisioterapia assistida por profissional, que objetivava medidas antiálgicas e anti-inflamatórias e ganho de ADM passiva e autopassiva, limitando-se a rotação externa à $30^{\circ}$ até a oitava semana de pós-operatório. A ADM completa foi recuperada após a décima segunda semana. Depois, iniciou-se o reforço muscular da cintura escapular, do manguito rotador, e da atividade proprioceptiva. Todas as atividades de impacto foram liberadas após o sexto mês de pós-operatório, desde que nas radiografias fosse identificado um bom posicionamento dos parafusos e consolidação do enxerto do coracoide.

\section{Coleta de Dados}

Por meio de um formulário, coletaram-se informações relativas ao sexo, idade, dominância, lado acometido, profissão, comorbidades, tabagismo, prática esportiva, complicações pós-operatórias (infecção, rigidez articular, soltura de implantes, recidivas), intensidade da dor segundo a Escala Visual Analógica (EVA), que teve que ser adaptada perguntando ao paciente a percepção da dor de 1 a 10, a percepção subjetiva da função pela escala Subjective Shoulder Value (SSV), e a pontuação funcional segundo The Western Ontario Shoulder Instability Index (WOSI).

\section{Análise Estatística}

A análise estatítica foi realizada por meio do teste $t$ de Student. O teste de Tukey foi empregado para a análise de comparações múltiplas usando análise de variância unidirecional (one-way analysis of variance [ANOVA], em inglês), para os dados paramétricos, e o teste de Kruskal-Wallis, para os dados não paramétricos.

A análise de correlação foi realizada com o teste de Spearman para dados não paramétricos.

Para a análise multivariada, foi realizado o teste de regressão linear, considerando como "variável resposta" cada um dos instrumentos utilizados (SSV, EVA, WOSI, SF12V2 PCS e SF-12V2 MCS). Após a análise bivariada, foram selecionadas para a análise multivariada aquelas variáveis que apresentaram valor de $p \leq 0,3$.

Em todos as análises realizadas, as diferenças obtidas foram consideradas estatisticamente significativas quando $p<0,05$.

As análises estatísticas foram realizadas utilizando os programas Prism (Graphpad Software, Inc., San Diego, CA, Estados Unidos), versão 5.0 para Windows, e Stata (Statacorp LLC, College Station, TX, Estdos Unidos), versão 14.0.

\section{Resultados}

A população do estudo foi composta exclusivamente por pacientes do sexo masculino, com média de idade de 26 $( \pm 5,17)$ anos, que variou de 18 a 35 anos. No total, $69,23 \%$ afirmaram realizar alguma atividade esportiva, sendo predominantemente recreativa. Os parâmetros analisados na - Tabela 1 não apresentaram diferença estatisticamente significativa na população estudada $(p>0,05)$.
A média de tempo entre a primeira luxação e o procedimento cirúrgico foi de 24 meses, com a ocorrência média de 10 luxações. A maioria $(84,62 \%)$ dos pacientes não apresentou episódios de recidiva da luxação. Ao todo, $3(11,54 \%)$ pacientes tiveram somente 1 recidiva, e 1 (3,85\%) paciente teve 2. No entanto, a recorrência ficou limitada a esses casos, e reoperações não foram necessárias. Em relação ao grau de satisfação, 92,31\% dos pacientes informaram estar satisfeitos, quando da consulta por telefonema, inclusive alguns dos que apresentaram recidiva (-Tabela $\mathbf{2}$ ).

\section{Análise Funcional}

A média da amostra na escala SSV foi de $90 \%$. $025^{\circ}$ percentil (P25) foi de $80 \%$, e o $75^{\circ}$ percentil (P75) foi de $100 \%$. A pontuação da maioria dos pacientes na escala SSV ficou entre $80 \%$ e $100 \%$ na escala SSV.

Na EVA, 76,92\% (20/26) dos pacientes relataram não sentir dor (0/10); para 7,69\% (2/26), a dor foi leve (2/10), e, para $15,38 \%$ (4/26) dos pacientes, foi moderada (3/10).

Segundo os dados gerais da escala WOSI, a classificação média dos pacientes foi de $18,70 \%$, com mínimo de $2,38 \%$ (melhor pontuação) e máximo de $45,24 \%$ (pior pontuação). Considerando os 4 domínios de forma isolada, os sintomas físicos apresentaram a melhor porcentagem $(8,5 \%)$, ao passo que o pior resultado foi observado quanto ao estilo de vida (20\%) (-Tabela 3).

Na avaliação pelo SF-12V2, o valor médio da pontuação do componente físicos (PCF) foi de 55,93 ( $\pm 3,44)$, e o da pontuação do compenente mental (PCM) foi de 45,22 ( $\pm 5,96)$. Considerando-se os valores padronizados para a população geral, de $50 \pm 10$, o PCF e o PCM estavam dentro da média de qualidade de vida ( - Tabela 4). Nesse âmbito, observou-se uma correlação positiva $(p<0,0001, r=0,7252)$ entre as pontuações na EVA e no WOSI. Assim, quanto melhor os resultados da EVA, melhor será a qualidade de vida do paciente medida pelo WOSI.

Observou-se também que as pontuações foram coerentes com a do PCF, que apresentou correlação negativa com a EVA e o WOSI ( $p=0,0066 ; r=-0,5189$; е $p=0,0159 ; r=-0,4681$, respectivamente). Com isso, quanto melhor o PCS, menores são as pontuações na EVA e no WOSI, o que demonstra melhor qualidade de vida.

Em relação aos pacientes que praticam algum esporte, os parâmetros do SSV ( $p=0,0081 ; r=0,5076$-correlação positiva) apresentaram melhora, ao passo que a correlação das pontuações na EVA ( $p=0,0124 ; r=-0,4832) \mathrm{e}$ no WOSI ( $p=0,0112 ; r=-0,4892$ ) foi negativa, porque, quanto melhor o valor destes, melhor a qualidade de vida.

A ocorrência de recidivas, estava inversamente relacionada ao SSV ( $p=0,0033 ; r=-0,5537)$, o que sugere uma pior avaliação subjetiva funcional pelo paciente.

O grau de satisfação dos pacientes em relação ao procedimento cirúrgico foi inversamente proporcional ao tempo entre a primeira luxação e a realização da cirurgia, ou seja, quanto menor o tempo, maior foi a satisfação $(p=0,0499$; $r=-0,3884$ ) ( - Tabela 5). 
Tabela 1 Características da amostra do estudo

\begin{tabular}{|c|c|c|c|}
\hline Idade (anos) & & & \\
\hline Média (desvio padrão) & $26( \pm 5,17)$ & & \\
\hline Mínimo-máximo & $18-35$ & & \\
\hline Sexo & Frequência absoluta & $\%$ & Valor de $p$ \\
\hline Masculino & 26 & 100,00 & $1,0000^{\top}$ \\
\hline Feminino & 0 & 0,00 & \\
\hline TOTAL & 26 & 100,00 & \\
\hline Comorbidades & Frequência absoluta & $\%$ & Valor de $p$ \\
\hline Sim & 1 & 3,85 & $1,0000^{\top}$ \\
\hline Não & 25 & 96,15 & \\
\hline TOTAL & 26 & 100,00 & \\
\hline Tabagismo & Frequência absoluta & $\%$ & Valor de $p$ \\
\hline Sim & 2 & 7,69 & $1,0000^{\top}$ \\
\hline Não & 24 & 92,31 & \\
\hline TOTAL & 26 & 100,00 & \\
\hline Prática de esportes & Frequência absoluta & $\%$ & Valor de $p$ \\
\hline Sim & 18 & 69,23 & $1,0000^{\top}$ \\
\hline Não & 8 & 30,77 & \\
\hline TOTAL & 26 & 100,00 & \\
\hline Tipo de atividade esportiva & Frequência absoluta & $\%$ & Valor de $p$ \\
\hline Academia & 3 & 16,67 & $0,8143^{\mathrm{TK}}$ \\
\hline Futebol & 11 & 61,11 & \\
\hline Trilha de moto & 2 & 11,11 & \\
\hline Musculação e mountain biking & 1 & 5,56 & \\
\hline Vôlei e futebol & 1 & 5,56 & \\
\hline TOTAL & 18 & 100,00 & \\
\hline Competitivo versus recreativo & Frequência absoluta & $\%$ & Valor de $p$ \\
\hline Competitivo & 0 & 0,00 & $1,0000^{\top}$ \\
\hline Recreativo & 18 & 100,00 & \\
\hline TOTAL & 18 & 100,00 & \\
\hline
\end{tabular}

Notas: ${ }^{\top} T e s t e ~ t$ de Student, utilizado para detectar a existência de diferenças significativas na população. ${ }^{\text {TK }}$ Teste de Tukey para comparações múltiplas, utilizando análise de variância unidirecional.

Tabela 2 Caracterização dos pacientes segundo a ocorrência de luxações

\begin{tabular}{|l|l|l|l|}
\hline \multicolumn{2}{|l|}{ Tempo entre a primeira luxação e o procedimento cirúrgico (meses) } & \multicolumn{2}{l|}{} \\
\hline Mediana & 24 & \\
\hline P25-P75 & $18-48$ & \\
\hline Mínimo-máximo & $6-96$ & \\
\hline Número de luxações antes do procedimento cirúrgico & \multicolumn{1}{l|}{} \\
\hline Mediana & 45 & & \\
\hline P25-P75 & $12-65$ & & \\
\hline Mínimo-máximo & $2-150$ & \\
\hline Recidiva & Frequência absoluta & $\%$ & Valor de $p$ \\
\hline Nenhuma & 22 & 84,62 & $1,0000^{\text {TK }}$ \\
\hline
\end{tabular}


Tabela 2 (Continued)

\begin{tabular}{|l|l|l|l|}
\hline Uma vez & 3 & 11,54 & \\
\hline Duas vezes & 1 & 3,85 & \\
\hline TOTAL & 26 & $\mathbf{1 0 0 , 0 0}$ & \\
\hline Reoperação & Frequência absoluta & $\%$ & Valor de $p$ \\
\hline Sim & 0 & 0,00 & $1,0000^{\top}$ \\
\hline Não & 26 & 100,00 & \\
\hline TOTAL & 26 & $\mathbf{1 0 0 , 0 0}$ & \\
\hline Satisfação & Frequência absoluta & $\%$ & Valor de $p$ \\
\hline Satisfeito & 24 & 92,31 & $1,0000^{\top}$ \\
\hline Não satisfeito & 2 & 7,69 & \\
\hline TOTAL & 26 & $\mathbf{1 0 0 , 0 0}$ & \\
\hline Realizou o procedimento de Bankart previamente ao de Latarjet? & Frequência absoluta & $\%$ & Valor de $p$ \\
\hline Sim & 3 & 11,54 & $1,0000^{\top}$ \\
\hline Não & 23 & 88,46 & \\
\hline TOTAL & 26 & $\mathbf{1 0 0 , 0 0}$ & \\
\hline
\end{tabular}

Abreviaturas: $\mathrm{P} 25,25^{\circ}$ percentil; $\mathrm{P} 75,75^{\circ}$ percentil.

Notas: ${ }^{\top}$ Teste $t$ de Student, utilizado para detectar a existência de diferenças significativas na população. ${ }^{\text {TK }}$ Teste de Tukey para comparações múltiplas, utilizando análise de variância unidirecional.

Tabela 3 Avaliação da qualidade de vida dos pacientes da amostra segundo a escala Subjective Shoulder Value (SSV)

\begin{tabular}{|c|c|c|c|}
\hline SSV - pontuação & Frequência absoluta & $\%$ & Valor de $p$ \\
\hline $100 \%$ & 10 & 38,46 & \multirow[t]{7}{*}{$1,0000^{\mathrm{KW}}$} \\
\hline $95 \%$ & 2 & 7,69 & \\
\hline $90 \%$ & 6 & 23,08 & \\
\hline $80 \%$ & 4 & 15,38 & \\
\hline $60 \%$ & 1 & 3,85 & \\
\hline $56 \%$ & 1 & 3,85 & \\
\hline $40 \%$ & 2 & 7,69 & \\
\hline TOTAL & 26 & 100,00 & \\
\hline \multirow[t]{2}{*}{ SSV (\%) - geral } & Mediana & P25-P75 & Mínimo-máximo \\
\hline & 90 & $80-100$ & $40-100$ \\
\hline
\end{tabular}

Abreviaturas: $\mathrm{P} 25,2^{\circ}$ percentil; $\mathrm{P} 75,75^{\circ}$ percentil.

Nota: ${ }^{\mathrm{KW}}$ Teste de Kruskal-Wallis, utilizado para detectar a existência de diferenças significativas na população (dados não paramétricos).

\section{Discussão}

O achado mais importante deste estudo foi a correlação entre a maior satisfação dos pacientes e a diminuição do tempo entre a luxação e o procedimento cirúrgico. Desta maneira, confirma-se a hipótese de que o tempo para a realização do procedimento cirúrgico influencia na satisfação do paciente.

A amostra apresentava predominantemente homens jovens, com média de idade de 26 anos, o que está de acordo com a literatura que demonstra ocorrência maior em atletas de contato, os quais pertencem ao grupo de risco. ${ }^{17,18}$ Neles, o tempo médio entre o procedimento cirúrgico e a primeira luxação foi de 24 meses, com uma ocorrência média de 10 luxações antes da decisão de se submeter ao procedimento.
Estudos de metanálise ${ }^{10,19}$ relatam entre $25 \%$ e $31 \%$ de complicações possíveis com o procedimento de Latarjet . Por outro lado, ao analisar somente a ocorrência de recidivas, os índices variam de $1 \%$ a 7,5\%. ${ }^{7,13,20,21}$ A única complicação relatada neste estudo foi a recidiva, que ocorreu em 4 dos 26 pacientes $(15,39 \%)$. Como o estudo foi feito por entrevista pelo telefone, os pacientes podem ter tido dificuldade em identificar outras complicações, ou até mesmo em compreender que o termo luxação refere-se à incongruência total da articulação glenoumeral.

Na avaliação das escalas funcionais e de qualidade de vida, mais de $75 \%$ dos pacientes apresentaram valores maiores de $80 \%$ no SSV, o que demonstra uma avaliação subjetiva alta da funcionalidade. Em relação à EVA, o resultado de 76,92\% da 
Fatores prognósticos da cirurgia de Latarjet Vilela et al. 981

Tabela 4 Avaliação da qualidade de vida dos pacientes da amostra segundo o instrumento SF-12V2

\begin{tabular}{|c|c|c|c|c|c|c|}
\hline \multirow[t]{2}{*}{ Domínios do SF-12V2 } & \multicolumn{6}{|c|}{ Frequência das alternativas (\%) } \\
\hline & 1 & 2 & 3 & 4 & 5 & TOTAL (\%) \\
\hline Capacidade funcional & - & 9,62 & 90,38 & * & * & 100,00 \\
\hline Aspecto físico & - & 3,85 & - & 3,85 & 92,30 & 100,00 \\
\hline Dor corporal & 53,85 & 46,15 & - & - & - & 100,00 \\
\hline Saúde geral & 7,70 & 76,92 & 15,38 & - & - & 100,00 \\
\hline Vitalidade & 11,54 & 69,23 & 19,23 & - & - & 100,00 \\
\hline Aspecto social & 7,69 & - & - & 7,69 & 84,62 & 100,00 \\
\hline Aspecto emocional & - & 3,85 & 25,00 & 21,15 & 50,00 & 100,00 \\
\hline Saúde mental & 11,54 & 34,61 & 11,54 & 19,23 & 23,08 & 100,00 \\
\hline Resumo dos componentes físico e mental & \multicolumn{2}{|l|}{ Média } & \multicolumn{2}{|c|}{ Desvio padrão } & \multicolumn{2}{|c|}{ Mínimo-máximo } \\
\hline Pontuação do componente físico & \multicolumn{2}{|l|}{55,93} & \multicolumn{2}{|l|}{ $\pm 3,44$} & \multicolumn{2}{|c|}{$48,22-61,65$} \\
\hline Pontuação do componente mental & \multicolumn{2}{|l|}{45,22} & \multicolumn{2}{|l|}{ $\pm 5,96$} & \multicolumn{2}{|c|}{$32,20-58,83$} \\
\hline
\end{tabular}

SF-12v2® Health Survey é uma medida prática, confiável e válida da saúde física e mental. Cada pontuação do domínio de saúde contribui para as pontuações do Resumo do Componente Físico (PCS) e do Resumo do Componente Mental (MCS).

Notas: * Alternativas não disponíveis no domínio. - Alternativas não selecionadas pelos pacientes.

Tabela 5 Análise de correlação (Spearman) entre os fatores dos pacientes e as escalas de avaliação da qualidade de vida

\begin{tabular}{|c|c|c|c|c|c|c|c|c|}
\hline & PCM FINAL & SSV & EVA & WOSI & IDADE & $\begin{array}{l}\text { PRÁTICA DE } \\
\text { ESPORTES }\end{array}$ & $\begin{array}{l}\text { NÚMERO DE } \\
\text { RELUXAÇÕES }\end{array}$ & $\begin{array}{l}\text { GRAU DE } \\
\text { SATISFAÇÃO }\end{array}$ \\
\hline \multicolumn{9}{|l|}{ PCF FINAL } \\
\hline valor de r & $-0,6260$ & 0,1105 & $-0,5189$ & $-0,4681$ & 0,0302 & 0,2335 & $-0,1190$ & 0,0963 \\
\hline valor de $p$ & $0,0006^{*}$ & 0,5910 & $0,0066^{*}$ & $0,0159^{*}$ & 0,8835 & 0,2509 & 0,5627 & 0,6398 \\
\hline \multicolumn{9}{|l|}{ PCM FINAL } \\
\hline valor de r & & $-0,3983$ & 0,5980 & 0,4660 & $-0,1412$ & $-0,3670$ & 0,0699 & $-0,0963$ \\
\hline valor de $p$ & & $0,0439^{*}$ & $0,0013^{*}$ & $0,0164^{*}$ & 0,4915 & 0,0652 & 0,7346 & 0,6398 \\
\hline \multicolumn{9}{|l|}{ SSV } \\
\hline valor de r & $-0,3983$ & & $-0,4245$ & $-0,5446$ & 0,1149 & 0,5076 & $-0,5537$ & 0,2798 \\
\hline valor de $p$ & $0,0439^{*}$ & & $0,0307^{*}$ & $0,0040^{*}$ & 0,5762 & $0,0081^{*}$ & $0,0033^{*}$ & 0,1663 \\
\hline \multicolumn{9}{|l|}{ EVA } \\
\hline valor de $r$ & 0,5980 & $-0,4245$ & & 0,7252 & $-0,3255$ & $-0,4832$ & 0,2875 & 0,1569 \\
\hline valor de $p$ & $0,0013^{*}$ & $0,0307^{*}$ & & $0,0000^{*}$ & 0,1047 & $0,0124^{*}$ & 0,1544 & 0,4440 \\
\hline \multicolumn{9}{|l|}{ WOSI } \\
\hline valor de $r$ & 0,4660 & $-0,5446$ & 0,7252 & & $-0,2988$ & $-0,4892$ & 0,3536 & $-0,2311$ \\
\hline valor de $p$ & $0,0164^{*}$ & $0,0040^{*}$ & $0,0000^{*}$ & & 0,1382 & $0,0112^{*}$ & 0,0764 & 0,2560 \\
\hline \multicolumn{9}{|l|}{ IDADE } \\
\hline valor de r & $-0,1412$ & 0,1149 & $-0,3255$ & $-0,2988$ & & 0,4684 & $-0,1034$ & $-0,2994$ \\
\hline valor de $p$ & 0,4915 & 0,5762 & 0,1047 & 0,1382 & & $0,0158^{*}$ & 0,6151 & 0,1373 \\
\hline \multicolumn{9}{|c|}{ PRÁTICA DE ESPORTES } \\
\hline valor de $r$ & $-0,3670$ & 0,5076 & $-0,4832$ & $-0,4892$ & 0,4684 & & $-0,1595$ & $-0,1925$ \\
\hline valor de $p$ & 0,0652 & $0,0081^{*}$ & $0,0124^{*}$ & $0,0112^{*}$ & $0,0158^{*}$ & & 0,4364 & 0,3462 \\
\hline \multicolumn{9}{|l|}{$\begin{array}{l}\text { NÚMERO DE } \\
\text { RECIDIVAS }\end{array}$} \\
\hline valor de r & 0,0699 & $-0,5537$ & 0,2875 & 0,3536 & $-0,1034$ & $-0,1595$ & & 0,1228 \\
\hline valor de $p$ & 0,7346 & $0,0033^{*}$ & 0,1544 & 0,0764 & 0,6151 & 0,4364 & & 0,5502 \\
\hline
\end{tabular}


982 Fatores prognósticos da cirurgia de Latarjet Vilela et al.

Tabela 5 (Continued)

\begin{tabular}{|c|l|l|l|l|l|l|l|l|}
\hline & PCM FINAL & SSV & EVA & WOSI & IDADE & $\begin{array}{l}\text { PRÁTICA DE } \\
\text { ESPORTES }\end{array}$ & $\begin{array}{l}\text { NÚMERO DE } \\
\text { RELUXAÇÕES }\end{array}$ & $\begin{array}{l}\text { GRAU DE } \\
\text { SATISFAÇÃO }\end{array}$ \\
\hline GRAU DE SATISFAÇÃO \\
\hline valor de $r$ & $-0,0963$ & 0,2798 & 0,1569 & $-0,2311$ & $-0,2994$ & $-0,1925$ & 0,1228 & \\
\hline valor de $p$ & 0,6398 & 0,1663 & 0,4440 & 0,2560 & 0,1373 & 0,3462 & 0,5502 & \\
\hline TEMPO (ENTRE A PRIMEIRA LUXAÇÃO E O PROCEDIMENTO CIRÚRGICO) \\
\hline valor de $r$ & 0,1409 & 0,2224 & 0,0131 & 0,2189 & 0,2304 & 0,3363 & $-0,2624$ & $-0,3884$ \\
\hline valor de $p$ & 0,4925 & 0,2748 & 0,9493 & 0,2828 & 0,2574 & 0,0930 & 0,1953 & $0,0499^{*}$ \\
\hline
\end{tabular}

Abreviaturas: EVA, Escala Visual Analógica; PCF, pontuação do componente físico; PCM, pontuação do componente mental; SSV, Subjective Shoulder Value; WOSI, The Western Ontario Shoulder Instability Index.

Nota: * Diferença significativa segundo a correlação de Spearman $(p<0,05)$.

amostra foi 0 , ou seja, nenhuma dor, e o pior foi 3, que equivale a dor de leve a moderada. Portanto, pode-se afirmar que ows resultdos relacionados à dor foram bastante satisfatórios. Ao se questionar sobre a satisfação, 92,31\% relataram estar satisfeitos, assim como no trabalho de Da Silva et al. $^{22}$ Taxas de satisfação > 90\% também foram encontradas por Hurley et al., ${ }^{4}$ mesmo após seguimento de 16 anos. Hovelius et al. ${ }^{23}$ acompanharam 118 ombros, e relataram taxas de satisfação de $98 \%$ após 15 anos. Schroder et al., ${ }^{24} \mathrm{em}$ um o trabalho envolvendo 49 pacientes com seguimento de 26,4 anos, encontraram taxa de cerca de $70 \%$ de resultados bons ou excelentes. Já Silva et al. ${ }^{25}$ obtiveram resultados bons e excelentes em $82,7 \%$ dos casos.

O SF-12V2 permite a avaliação e a comparação com o valor padronizado para a população geral: $50 \pm 10$. Portanto, podese afirmar que a amostra do presente estudo estava próxima dessa faixa, pois os valor médio encontrado para o PCF foi de 55,93, e, para o PCM, de 45,22. Esses valores também demonstram que, com o procedimento de Latarjet, o paciente pode voltar a níveis similares a aos de um indivíduo que nunca apresentou luxação quanto às avaliações física e mental.

Na análise da correlação entre as diferentes variáveis, foi observada coerência entre as escalas subjetivas: o SSV demonstrou estar inversamente relacionado tanto à EVA quanto ao WOSI, o que confirma que, quanto mais próxima for a avaliação subjetiva do ombro operado em relação a um ombro saudável, menor a sensação de dor e limitação funcional.

$\mathrm{O}$ número de recidivas piorou as pontuações no SSV segundo a correlação de Spearman $(p=0,0033, \mathrm{r}=0,5537)$, o que sugere que recidivas acarretam piores resultados funcionais. Além disso, um número maior de luxações préoperatórias influenciou negativamente nos resultados do EVA. A cada luxação, pode ocorrer um aumento da lesão dos estabilizadores estáticos e dinâmicos, bem como do tamanho das lesões de Hill-Sachs e de Bankart.

Por outro lado, a prática de esporte demonstrou-se benéfica para a qualidade de vida, e apresentou, pela correlação de Spearman, as melhores pontuações no SSV $(p=0,008)$, na EVA e no WOSI, o que foi reiterado na análise multivariada da EVA. A importância do retorno ao esporte foi reforçada por
Warth et al., ${ }^{26}$ que relataram que umas das grandes preocupações do paciente no pré-operatório é a capacidade de retornar ao esporte.

Pela correlação de Spearman, demonstrou-se que a satisfação do paciente foi inversamente proporcional ao tempo entre a primeira luxação e a realização da cirurgia. Dessa maneira, a indicação cirúrgica no menor tempo possível pode ser benéfica para o paciente em termos dos resultados subjetivos.

O presente trabalho apresenta limitações por ser retrospectivo, e por ter sido realizado pelo telefone. Portanto, nele pode ter havido um viés quanto à transmissão de informação e às complicações referentes ao exame físico, como a limitação da ADM, que não foi avaliada. A amostra de 26 pacientes é pequena, e limita a avaliação estatística. $O$ tempo de seguimento de 12 meses também é considerado relativamente curto para tratamentos cirúrgicos e instabilidade recorrente do ombro.

\section{Conclusão}

Fatores como um tempo longo entre o primeiro episódio de luxação e a realização do procedimento cirúrgico e uma ocorrência maior de recidivas influenciaram negativamente na qualidade de vida dos pacientes.

A indicação precoce do tratamento cirúrgico da instabilidade anterior glenoumeral pode proporcionar melhores resultados funcionais subjetivos ao paciente.

\section{Fontes de Financiamento}

Esta pesquisa não recebeu nenhuma bolsa específica de agências de fomento nos setores público, comercial, ou sem fins lucrativos.

Conflito de Interesses

Os autores declaram não haver conflitos de interesse.

\section{Referências}

1 Rowe CR, Patel D, Southmayd WW. The Bankart procedure: a longterm end-result study. J Bone Joint Surg Am 1978;60(01):1-16

2 Itoi E, Lee SB, Berglund LJ, Berge LL, An KN. The effect of a glenoid defect on anteroinferior stability of the shoulder after Bankart 
repair: a cadaveric study. J Bone Joint Surg Am 2000;82(01): 35-46

3 Latarjet M. [Treatment of recurrent dislocation of the shoulder]. Lyon Chir 1954;49(08):994-997

4 Hurley ET, Jamal MS, Ali ZS, Montgomery C, Pauzenberger L, Mullett $\mathrm{H}$. Long-term outcomes of the Latarjet procedure for anterior shoulder instability: a systematic review of studies at 10-year follow-up. J Shoulder Elbow Surg 2019;28(02):e33-e39

5 Metais P, Clavert P, Barth J, et al French Arthroscopic Society. Preliminary clinical outcomes of Latarjet-Patte coracoid transfer by arthroscopy vs. open surgery: Prospective multicentre study of 390 cases. Orthop Traumatol Surg Res 2016;102(8S):S271-S276

6 An VVG, Sivakumar BS, Phan K, Trantalis J. A systematic review and meta-analysis of clinical and patient-reported outcomes following two procedures for recurrent traumatic anterior instability of the shoulder: Latarjet procedure vs. Bankart repair. J Shoulder Elbow Surg 2016;25(05):853-863

7 Hovelius L, Sandström B, Olofsson A, Svensson O, Rahme H. The effect of capsular repair, bone block healing, and position on the results of the Bristow-Latarjet procedure (study III): long-term follow-up in 319 shoulders. J Shoulder Elbow Surg 2012;21(05): 647-660

8 Hovelius L, Vikerfors O, Olofsson A, Svensson O, Rahme H. Bristow-Latarjet and Bankart: a comparative study of shoulder stabilization in 185 shoulders during a seventeen-year follow-up. J Shoulder Elbow Surg 2011;20(07):1095-1101

9 Bliven KCH, Parr GP. Outcomes of the Latarjet Procedure Compared With Bankart Repair for Recurrent Traumatic Anterior Shoulder Instability. J Athl Train 2018;53(02):181-183

10 Delaney RA, Freehill MT, Janfaza DR, Vlassakov KV, Higgins LD, Warner JJP. 2014 Neer Award Paper: neuromonitoring the Latarjet procedure. J Shoulder Elbow Surg 2014;23(10):1473-1480

11 Griesser MJ, Harris JD, McCoy BW, et al. Complications and reoperations after Bristow-Latarjet shoulder stabilization: a systematic review. J Shoulder Elbow Surg 2013;22(02):286-292

12 Gupta A, Delaney R, Petkin K, Lafosse L. Complications of the Latarjet procedure. Curr Rev Musculoskelet Med 2015;8(01): 59-66

13 Bessière C, Trojani C, Carles M, Mehta SS, Boileau P. The open latarjet procedure is more reliable in terms of shoulder stability than arthroscopic bankart repair. Clin Orthop Relat Res 2014;472 (08):2345-2351

14 Cho NS, Yoo JH, Rhee YG. Management of an engaging Hill-Sachs lesion: arthroscopic remplissage with Bankart repair versus
Latarjet procedure. Knee Surg Sports Traumatol Arthrosc 2016; 24(12):3793-3800

15 Russo R, Della Rotonda G, Cautiero F, et al. Arthroscopic Bankart repair associated with subscapularis augmentation (ASA) versus open Latarjet to treat recurrent anterior shoulder instability with moderate glenoid bone loss: clinical comparison of two series. Musculoskelet Surg 2017;101(01):75-83

16 McHale KJ, Sanchez G, Lavery KP, et al. Latarjet Technique for Treatment of Anterior Shoulder Instability With Glenoid Bone Loss. Arthrosc Tech 2017;6(03):e791-e799

17 Galvin JW, Ernat JJ, Waterman BR, Stadecker MJ, Parada SA. The Epidemiology and Natural History of Anterior Shoulder Instability. Curr Rev Musculoskelet Med 2017;10(04):411-424

18 Waterman B, Owens BD, Tokish JM. Anterior Shoulder Instability in the Military Athlete. Sports Health 2016;8(06):514-519

19 Shah AA, Butler RB, Romanowski J, Goel D, Karadagli D, Warner JJ. Short-term complications of the Latarjet procedure. J Bone Joint Surg Am 2012;94(06):495-501

20 Young AA, Maia R, Berhouet J, Walch G. Open Latarjet procedure for management of bone loss in anterior instability of the glenohumeral joint. J Shoulder Elbow Surg 2011;20(2, Suppl)S61-S69

21 de Beer JF, Roberts C. Glenoid bone defects-open latarjet with congruent arc modification. Orthop Clin North Am 2010;41(03): 407-415

22 da Silva LA, da Costa Lima ÁG, Kautsky RM, Santos PD, do Val Sella $\mathrm{G}$, Checchia SL. Evaluation of the results and complications of the Latarjet procedure for recurrent anterior dislocation of the shoulder. Rev Bras Ortop 2015;50(06):652-659

23 Hovelius L, Sandström B, Sundgren K, Saebö M One hundred eighteen Bristow-Latarjet repairs for recurrent anterior dislocation of the shoulder prospectively followed for fifteen years: study Iclinical results. J Shoulder Elbow Surg 2004;13(05):509-516

24 Schroder DT, Provencher MT, Mologne TS, Muldoon MP, Cox JS. The modified Bristow procedure for anterior shoulder instability: 26-year outcomes in Naval Academy midshipmen. Am J Sports Med 2006;34(05):778-786

25 Silva LA, Lima AG, Kautsky RM, Santos PD, Sella GV, Checchia SL. Avaliação dos resultados e das complicações em pacientes com instabilidade anterior do ombro tratados pela técnica de Latarjet. Rev Bras Ortop 2015;50(06):652-659

26 Warth RJ, Briggs KK, Dornan GJ, Horan MP, Millett PJ. Patient expectations before arthroscopic shoulder surgery: correlation with patients' reasons for seeking treatment. J Shoulder Elbow Surg 2013;22(12):1676-1681 